\title{
The Status of Knowledge Management in Teaching Hospitals Affiliated to Tehran Universities of Medical Sciences
}

\author{
Rafat MohebbiFar ${ }^{1}$, Parisa Ghaboosi ${ }^{2}$, Hasan Jahani hashemi ${ }^{3}$, Fatemeh Akbari Rad $^{4}$, Mohammad Mohseni ${ }^{5} \&$ \\ Meysam Safi Keykale ${ }^{6}$ \\ ${ }^{1}$ Department of Health Management, School of Public Health, Qazvin University of Medical Sciences, Qazvin, \\ Iran \\ ${ }^{2}$ School of Public Health, Qazvin University of Medical Sciences, Qazvin, Iran \\ ${ }^{3}$ Department of Biostatistics, School of Medicine, Qazvin University of Medical Sciences, Qazvin, Iran \\ ${ }^{4}$ Research Center for Modeling in Health, Institute for Futures Studies in Health, Kerman University of Medical \\ Sciences, Kerman, Iran \\ ${ }^{5}$ Health Management And Economics Research Center, Iran University of Medical Sciences, Tehran, Iran \\ ${ }^{6}$ Research Center for Health Services Management, Institute for Futures Studies in Health, Kerman University \\ of Medical Sciences, Kerman, Iran \\ Correspondence: Mohammad Mohseni, Health Management and Economics Research Center, Iran University of \\ Medical Sciences, Tehran, Iran. Tel: 98-937-864-2255. E-mail: mmohseni1986@gmail.com
}

Received: July 25, 2013 Accepted: August 12, 2014 Online Published: September 29, 2014

doi:10.5539/ass.v10n19p191 URL: http://dx.doi.org/10.5539/ass.v10n19p191

\begin{abstract}
Knowledge management is one of the modern management approaches, which has become the key to success in organizations. For this reason, the purpose of the present paper is to investigate the status of knowledge management in education and treatment centers of Tehran Universities of Medical Sciences. This study was a descriptive-analytical survey. The statistical population included all the staffs of hospitals of Tehran Universities of Medical Sciences. Sample size was estimated 502 persons using random sampling method. Data collection tool is Probst's knowledge management questionnaire. Reliability of the questionnaire was obtained 0.89 using Cronbach's alpha, and its validity has been confirmed by experienced professors. In order to analyze the data using SPSS software, T test, ANOVA test, correlation coefficient, and regression were used. According to the obtained data, it was found that the status of knowledge management and its dimensions (Goals, identification, acquisition, development, distribution, use, preservation, and knowledge measurement) in the studied hospitals was at an average level. Considering the responsibility of teaching and treatment centers in educating students, more attention should be paid to knowledge management in these organizations and it should be prioritized. Therefore, knowing their status in the scope of knowledge management, the authorities of the hospitals should focus on all dimensions so as to reduce the interval between the status quo and the desired status.
\end{abstract}

Keywords: knowledge management, teaching hospital, Tehran

\section{Introduction}

In today's world, the competitive conditions and environments of many organizations are very complicated and variable and more extensive than what it was in the past. This pace is significantly more than the response time and the capacity to adapt to the new environment (Niroomand \& Zare, 2008). Alvin Toffler believes that entering into the 21 st century as the meta-theory era will attract people if human beings can bear the burden of its changes and accept them courageously. This entails having knowledge, skill, insight, and dynamism (Sadeghi, Jafari, Khodayari, Pakdaman, Mohammadi \& Ahadinezhad, 2011). Drucker (1999) states that if in the 20th century, the most valuable asset of a company was its production facilities, in the 21 st century, knowledge workers and their efficiency are considered the most important and the most valuable asset of companies (Huang \& Chen, 2009).

Other pioneer theorists in the field of organization and management believe that investment of a company on knowledge is more profitable than that on its components (Nonaka, Takeuchi \& Umemoto, 1996). Organizations 
need knowledge management so as to be able to survive in the competitions, adapt to the changes of the creative environment, respond to the market demands, enhance individuals and their capacity, and maintain good relations with suppliers, customers, and partners (Edmonds, 2002). In fact, knowledge management is one of the modern approaches of management which has turned into the key to success in organizations (Pour Asghar, 2008).

In many of the organizations and institutions, one of the essential problems is that there is not adequate awareness about individuals' wisdom and knowledge. Effective management of knowledge, by focusing on the solutions that encompass the whole system i.e. the organization, human resources, and technology, is one of the most important tools for solving this problem (RafatiShaldehi, Hasnavi, Beh Azin \& BaniTaba, 2008). The more knowledge we have of a field, the more capacity we have for performing jobs well (Jones, 2007).

Many definitions have been suggested for knowledge management among which we can refer to the following ones: knowledge management is a scientific discipline which encourages and reinforces the method of mutual support for creating, grabbing, organizing, and using information(Haney, 2003); knowledge management is defined as a set of ordered and systematic organizational activities which are accomplished through the available knowledge in order to achieve a more important goal, and the available knowledge includes all the experiences and lessons of an organization's staff and all the internal documents and reports of an organization (Merwik, 2001); knowledge management is defined as utilization and development of knowledge assets of an organization for the sake of realizing the objectives (PourAsghar, 2008).

Many authors have so far presented models of knowledge management process, most of which are almost the same in terms of content, but have different terms and ordered phases (Afrazeh, 2007).

Knowledge advisory group of KPMG Institution (2003) conducted an extensive research on knowledge management among 500 organizations in Britain, France, Germany and Netherlands. The research showed that investing on knowledge management will have huge economic profits (Geyer, 2001). Evaluating the level of knowledge management in the University of Police Sciences, Hashemi (2010) concluded that knowledge management level in this university is far away from the desired status. Moreover, by imparting the general policies of the administrative system by the Supreme Leader (March, 2010) and according to the notification of the Armed Forces General Staff about knowledge management, as well as the focusing of the government on creating a knowledge society and preferring a movement towards knowledge-oriented economies in the 20 -year vision of the country and the fourth and fifth programs of development, the important matter that has aroused is that how should we exploit knowledge as an important source of competitive advantage (Tavallaei, 2009). On one hand, the Department of Health as an organization which needs skillful and knowledgeable staffs and is tightly related to the society's health, requires using efficient methods of offering services for improving the quality, reducing health costs, and meeting the clients' needs timely. This is possible only through using modern methods of information management and allocating proper time to knowledge management (Mirghafoori, Nejad, $\&$ Arani, 2010). Therefore, it is necessary that the managers of health-care and treatment services consider the job and the staffs that offer these services as the means of generating knowledge (Maleki, Panah, \& Gohari, 2009).

According to the above reasons, in this paper we attempted to study the status of knowledge management in education and treatment centers of Medical Sciences Universities of Tehran, Shahid Beheshti, and Iran, so that managers of hospitals, who intend to have widespread and successful development and acquire competitive advantage, utilize its results and correspondingly equip their staffs with knowledge as the most important organizational capital and thereby move effectively towards the growth, development, and realization of their objectives.

\section{Method}

This research is a descriptive-analytical survey which has been conducted in 2013-14. Data collection tool was Probst et al.'s knowledge management questionnaire which, using 21 questions, has measured 8 dimensions of knowledge management (Goals, identification, acquisition, development, distribution, use, preservation, and knowledge measurement) (Probst, Raub, \& Romhardt, 2000). Likert's five-point scale has been used to answer the questions. Reliability of the questionnaire is determined by Cronbach's alpha as 0.89 . Its validity has been confirmed by the comments and judgments of the experienced and qualified professors.

The studied population of this research includes all the staffs working in treatment, paraclinic, and administrative groups of education and treatment centers of Tehran Universities of Medical Sciences, among which 9 education and treatment centers related to Medical Sciences Universities of Tehran, Iran, and Shahid Beheshti ( 3 hospitals from each university) were selected as the sample according to their capacity for work, their facilities, and based 
on having the highest number of beds in the centers. The selected hospitals are Imam Khomeini, Valy-e Asr and Sina hospitals which are related to Tehran University of Medical Sciences, Hashemi Nezhad, Rasool-e Akram and Firoozgar hospitals which are related to Iran University of Medical Sciences, and Ayat Allah Taleghani, Imam Hossein and Loghman-e Hakim hospitals which are related to Shahid Beheshti University of Medical Sciences.

The obtained sample size was estimated 502 persons. The share of each hospital from the total 502 questionnaires was determined by visiting the hospitals and identifying the total number of staffs and it was specified proportional to their size.

In analyzing the data using SPSS software, in addition to using descriptive statistical methods, $t$ tests were used to compare the mean of the two groups, ANOVA test was used to compare the mean of several groups, and correlation coefficient and regression were used to consider the relationship between quantitative variables.

\section{Results}

In this paper, after conducting the required studies, it was concluded that the majority and minority of the employed individuals in the studied hospitals according to the demographic features are as follows:

According to gender, majority of $74.6 \%$ are females and minority of $25.4 \%$ are males. According to job type, majority of $45.4 \%$ are working in treatment department and minority of $23.5 \%$ are working in paraclinical departments. According to age, $40.90 \%$ are in 30-40 age range and $22.1 \%$ are over 40 . According to years of service, $54.1 \%$ have job experiences less than 10 years and $15.1 \%$ have more than 20 years of job experience. According to educational background, 60\% have bachelor degree and 18.8\% own $\mathrm{PhD}$.

\subsection{The First Hypothesis}

Knowledge management of teaching hospitals affiliated to tehran, iran, and shahid beheshti universities of medical sciences, is not the same.

Table 1. Knowledge management score, sorted by university

\begin{tabular}{ccccc}
\hline Variable & University of Medical Sciences & Mean & Standard deviation & Sig \\
\hline & Tehran & 60.47 & 10.908 \\
Knowledge & Shahid Beheshti & 59.86 & 12.403 & 0.149 \\
management & Iran & 62.61 & 13.385 & 12.194 \\
\hline
\end{tabular}

Considering the significance level, it can be said that there was no significant difference between the mean score of knowledge management in different universities $(p=0.149)$. It means that knowledge management in the hospitals affiliated with different universities is almost the same (Table1).

\subsection{The Second Hypothesis}

There is a relationship between knowledge management and individuals' age.

Correlation coefficient between knowledge management and age was $r=-0.056$ ( $p$-value $=0.236$ ), which shows that there is no significant relationship between knowledge management and age. However, correlation coefficient between "knowledge acquisition" component and age was $r=-0.108$ ( $p$-value $=0.022$ ), which shows the existence of a significant relationship.

\subsection{The Third Hypothesis}

There is a relationship between knowledge management and gender.

Table 2. The relationship between knowledge management and gender

\begin{tabular}{ccccc}
\hline Variables & Gender & Mean & Standard deviation & Sig \\
\hline Knowledge & Male & 63.47 & 13.251 & 0.008 \\
management & Female & 59.95 & 11.699 & \\
\hline
\end{tabular}


Table 2 shows that there is a significant relationship between knowledge management and gender $(\mathrm{p}=0.008)$, and since the mean score of knowledge management of males is more than females, knowledge management of men is more than women.

\subsection{The Fourth Hypothesis}

There is a relationship between knowledge management and work experience.

By doing the ANOVA test, the significance level between knowledge management and work experience was obtained as $\mathrm{p}=0.289$, which shows that there is no relationship between these two components. The only significant relationship was obtained between "knowledge acquisition" component and individuals' work experience $(\mathrm{p}=0.009)$. By doing the post hoc Tukey test, it was found that the more work experience individuals obtain, the less knowledge they will acquire.

\subsection{The Fifth Hypothesis}

There is a relationship between knowledge management and educational degree.

Results of the ANOVA test showed that there is no significant relationship between knowledge management and the years of education $(\mathrm{p}=0.289)$.

\subsection{The Sixth Hypothesis}

There is a relationship between knowledge management and job rank.

By doing the ANOVA test, the significance level between knowledge management and job ranks (the clinical, administrative, and paraclinical group) was obtained as $\mathrm{p}=0.390$, which indicates that there is no relationship between the two.

Table 3. Mean and standard deviation of knowledge management and its dimensions

\begin{tabular}{lll}
\hline Variables & Standard deviation \pm mean & Mean (based on 100) \\
\hline Knowledge management & $60.84 \pm 12.19$ & 57.94 \\
Determining the knowledge goals & $6.13 \pm 1.54$ & 61.30 \\
knowledge identification & $9.05 \pm 2.02$ & 60.33 \\
knowledge acquisition & $5.31 \pm 1.74$ & 53.10 \\
knowledge development & $8.29 \pm 2.28$ & 55.26 \\
knowledge distribution & $12.28 \pm 2.90$ & 61.40 \\
knowledge use & $5.14 \pm 1.90$ & 51.40 \\
knowledge preservation & $9.06 \pm 2.25$ & 60.40 \\
knowledge measurement & $5.58 \pm 1.96$ & 55.80 \\
\hline
\end{tabular}

In Table 3, we obtain the mean of all variables by 100 so that they can be compared. The score $0-35$ shows low level, the score 35-65 shows the average level and the score 65-100 shows the desired level of the variables. Therefore, it can be said that the status of knowledge management and its dimensions is at an average level. Among the dimensions, "specifying the objectives" and "sharing the knowledge" have the highest score and "using the knowledge" has the lowest score.

\section{Discussion}

As it was mentioned before, the main purpose of this study is to investigate knowledge management in hospitals of Tehran Universities of Medical Sciences, and results show that the current status of knowledge management and each of its dimensions in all studied hospitals are at an average level. Mirghafoor, Farhang Nejad \& Sadeghi Arani (2010) assessed the performance of health and treatment sector in Yazd province with regard to knowledge management. They state that knowledge management is in an undesired status, and also from the range of the studied processes (strategy and goals, acquisition and creation, preservation and documentation, distribution, utilization, and knowledge measurement), only the utilization of knowledge was higher than the average level and was in a desired status. Also Griffith University (2002) conducted a research entitled as "Knowledge Management" on 10 Indian companies and concluded that the dimensions of knowledge management such as education and knowledge creation in these companies were less than the average level. 
Also Hashemi (2010) estimates the status of each dimension of knowledge management as less than the average level in Police Sciences University.

In some parts of this research it has been shown that knowledge management in men is higher than women. This is in line with Pohlman's results (Pohlman, 1996). However, it is not similar to Nir and Jokar's results which show that there is no relationship between knowledge and gender (Nayer \& Jookar, 2010). The result of the present study shows that there is no significant relationship between knowledge management and education, while Yousefi and Gholami (2006) found a significant relationship between education and knowledge management among teachers of Esfahan Education Organization.

Lack of significant relationship between individuals' working years and knowledge management which is obtained in this research is in line with Nir and Jokar's study (Nayer \& Jookar, 2010). In this research, it has been observed that by increasing individuals' career profile (working years), their knowledge acquisition is decreased and it is because by increasing individuals' service length and subsequently their experience and awareness in the organization, their motivation for achieving new knowledge become less and instead this motivation increases for participating in decision-makings and sharing experience with others. On the other hand, according to our cultural values and beliefs, the experienced people are more respected and their opinions are paid more attention. Also in this research, like Yaghoubi, Javadi \& Agharahimi study (2011), we concluded that there is no relationship between knowledge management and age. On the other hand, by increasing age, knowledge acquisition is decreased which can be due to the decrease in motivation and mood of the old people for obtaining knowledge.

\section{Conclusion}

As the results of this research show, the status of knowledge management in hospitals of Tehran Universities of Medical Sciences is assessed at an average level which needs more attention and precedence of knowledge management in work plans and goals than other organizations regarding its distance from the desired level as well as the role of these education and treatment centers in students' education. In addition, among the dimensions, using the knowledge has the lowest score which means that managers do no encourage their staff enough to use and apply new methods and knowledge, and people are not that much free to express their ideas and opinions; therefore, their motivation for learning techniques leading to faster and better efficiency is decreased and while all organizations are quickly progressing and moving to stay in a dynamic environment, this reluctance and laxation is spreading between staffs, and in long term leads the organization to stagnation and being distant from its goals; this can be an alarm for paying more attention to this dimension of knowledge management by hospital managers. Of course, this never means that other dimensions should not be taken into account since their status is not good either! Increasing the score of each dimension leads to the total increase of knowledge management. Therefore, it is necessary that hospital administers consider their position toward knowledge management, pay more attention to removing their weak points, and decrease the distance between the status quo and the desired status so as to improve knowledge management by improving each of them. They can use the following recommendations to achieve this goal.

\section{Recommendations:}

- Information should flow freely throughout the organization. By creating good relations between personnel and managers or in other words performing open doors policies, ideas, news and information become available for all staffs and ambiguity is avoided in the organization.

- Creating conversation room and some softwares for information exchange through question and answer and holding scientific seminars for conveying the experiences of the successful and retired staffs to others because one of the barriers of knowledge management development is lack of negotiation spaces and learning and talking culture in organizations. Although the underlying technical facilities and the knowledgeable and experienced people in different fields are abundant in hospitals, most of the people and staffs exchange their knowledge in the organization aisle which may not lead to the acquisition of their target knowledge after they had wasted their time.

- Designing and installing knowledge map in the organization in order to determine which knowledge is hold by which person? How can it be used? And also providing catalogs, brochures and billboards to present the last changes in the organization and installing them in crowded places such as the staff card system, self-services, sector entrances, etc.

- Holding learning courses not only for presenting theory issues and giving certificate of participation, but also for considering organization's needs, and therefore training the staff about the new tools and 
methods of practically preforming works. This should be in a way that staffs are encouraged to apply their learned knowledge using a strong assessment system and giving feedbacks.

- Providing a culture based on the trust between staffs and managers through reinforcing the friendly and effective relations so that people can express their ideas and thoughts without any fear and concern of being humiliated by other staffs.

- Creating a new role called "knowledge manager" in the organization for the sake of informing, considering the affairs, and creating infrastructures related to the processes of knowledge management.

Finally, it is once again stressed that knowledge is the principle factor in each organization of today's world and its management is also very important and vital; therefore, considering each of its dimensions is a critical issue. However, since knowledge management is not in its real position as a scientific field, there are issues and ambiguities about it, but the experiences of the developed and industrial countries show that the topic of knowledge management will be expressed as an inseparable part of the organizations in the future, and if organizations cannot prepare necessary infrastructures for implementing knowledge management, they will surely face different challenges.

\section{Acknowledgments}

This study was part of a M.S dissertation supported by Qazvin University of Medical Sciences. We want to thank all officials and staff of studied hospital for their sincere cooperation.

\section{References}

Niroomand, A., \& Zare, F. (2008). Survey of position of knowledge management based on Nonaka's theory and its relationship to organizational culture. Bojnourd branch, Islamic Azad University. Cultural Research, 4(14), 80-96.

Sadeghi, A., Jafari, H., Khodayari, R., Pakdaman, M., Mohammadi, R., \& Ahadi nezhad, B. (2011). A case study: The association between organizational culture with knowledge management in Hasheminezhad hospital Tehran. Hospital Journal, 10(2), 2-8.

Huang, H. T., \& Chen, Ch. (2009). Emerging organizational structure for knowledge-oriented team work using genetic algorithm. Expert System with Applications, 36(10), 12137-12142. http://dx.doi.org/10.1016/j.eswa. 2009.03.062

Nonaka, I., Takeuchi, H., \& Umemoto, K. (1996). A theory of organizational knowledge creation. International Journal of Technology Management, 11(7), 833-845.

Edmonds, E. (2002). Creativity art practice and knowledge. Comm of the ACM, 45(10), 91-95. http://dx.doi.org/10.1145/570907.570939

Pour Asghar, A. (2008). Knowledge management, Creativity \& Innovation. Tosse'e Saderat Journal, 11(70), 19-22.

Rafati, S. H., Hasnavi, R., BehAzin, F., \& BaniTaba, A. (2008). Survey Pattern of knowledge management in a military research center. Journal of Military Medicine, 10(3), 237-252.

Jones, M. (2007). Knowledge management and creativity: A technology-facilitated balance (Thesis). Polytechnic university. New York, USA.

Haney, D. S. (2003). Knowledge Management in a Professional Service Firm (Doctoral Dissertation). Indiana University. Proust Information and Learning Company.

Merwik, A. D. (2001). Knowledge management technology. Systems journal, 40(4), 814.

Afrazeh, A. (2007). Knowledge management (concepts, models, measurement and implementation) (2nd ed.). Tehran: Amir Kabir University.

Probst, G., Raub, S., \& Romhardt, K. (2000). Managing Knowledge: Building Blocks for Success. John Wiley \& Sons: New York.

Geyer, G. (2001). Tacit knowledge in organization. London: Sage publications.

Hashemi, S. (2010). Evaluation of Knowledge Management in University of Disciplinary Science. Disciplinary Management Studies Journal, 5(2), 183-214.

Tavallaei, R. (2009). Necessities and functions of knowledge management in cultural organizations. Journal of Political organization. 
Mirghafoori, S. H., Farhangnejad, M. A., \& SadeghiArani, Z. (2010). Performance Evaluation of Yazd's Health Sector on Applying Knowledge Management Process. Journal of Health Administration, 13(39), 79-88.

Maleki, M. R., Fateh Panah, A., \& Gohari, M. R. (2009). Evaluation of performance of Hasheminezhad hospital based on analysis \& Knowledge management \& pattern of Health Malcom Baldrige. Health Information Management Journal, 8(4), 580-586.

Griffith University. (2002). Knowledge management. Research report India, 20(5), 15-17.

Pohlman, L. (1996). Creativity, Gender and the Family: A Study of Creative Writers. The Journal of Creative Behavior, 30(1), 1-24. http://dx.doi.org/10.1002/j.2162-6057.1996.tb00754.x

Nayer, N., \& Jookar, A. (2012). The Relationship between Knowledge Management and Creativity among the Librarians of Shiraz University Libraries. Health Information Management Journal, 9(2), 224-232.

Yousefi, A., \& Gholami, B. (2006). View of female teachers about levels of Knowledge Management. Journal of Knowledge \& Research in Cultural Sciences, 1(10), 29-46.

Yaghoubi, M., Javadi, M., \& Agharahimi, Z. (2011). The Relationship between Knowledge Management and Demographic Features of Students in Isfahan University of Medical Sciences. Education in Medical Sciences Journal, 10(5), 831-838.

\section{Copyrights}

Copyright for this article is retained by the author(s), with first publication rights granted to the journal.

This is an open-access article distributed under the terms and conditions of the Creative Commons Attribution license (http://creativecommons.org/licenses/by/3.0/). 